ReVista del instituto de Ciencias JuRÍdicas
DE PUEBLA, MÉXICO, ISSN: $1870-2147$. AÑO VI NO PUEBLA, MÉXICO, ISSN: 1870-2147. AÑO VI
NO, JULIO-DICIEMBRE DE 2012, PP. 204-214

\title{
Estado anómico y protesta juvenil en las elecciones de $2012^{*}$ \\ Anomic State and the protest of young people in the 2012 elections
}

\author{
Xóchitl Patricia Campos López** \\ Diego Martín Velázquez Caballero****
}

\section{RESUMEN}

La perspectiva de los jóvenes universitarios tomará la delantera, si no en el gobierno, en el ámbito académico y de la opinión pública libre. Los universitarios encarnan fidedignamente las legitimas aspiraciones de un gran sector social capaz de instrumentar los cambios que reclama con presteza la consolidación democrática; esto es, una democracia creadora de las condiciones necesarias para el despegue de la clase media, no de cuanto imponga, como siempre, dicha componenda entre la partidocracia y los poderes fácticos, entre el corporativismo y el clientelismo.

PALABRAS CLAVE: Estado anómico, protesta juvenil, universidad, universitarios, estudiantes, elecciones, México, democracia, internet, redes sociales.

\begin{abstract}
The perspective of university students will take the lead, if not in the government, in the academia and in the freedom of public opinion. The university students faithfully embody the legitimate aspirations of a large social sector capable of implementing changes swiftly claiming democratic consolidation, that is, a democracy creating the necessary conditions for the takeoff of the middle class, not as imposing as always, this compromise between the party-and the powers that be, between corporatism and clientelism.
\end{abstract}

KEY WORDS: Anomic State, young people protest, college, university, students, elections, Mexico, democracy, internet, social networking.

* Recibido: 22 de agosto de 2012. Aceptado: 30 de septiembre de 2012.

* Profesora en la Facultad de Derecho y Ciencias Sociales de la Benemérita Universidad Autónoma de Puebla, México (campospaty@yahoo.com).

*** Profesor en la Facultad de Derecho y Ciencias Sociales de la Benemérita Universidad Autónoma de Puebla, México (golda21@hotmail.com). 


\section{Sumario}

1. Introducción

2. Los estudiantes universitarios como sujetos de clase media

3. Los estudiantes universitarios y sus preferencias electorales (el caso de la BUAP)

4. Internet como instrumento de liberación

5. A manera de conclusión

\section{Introducción*}

La historia de México, en su mayoría, se distingue por años de autoritarismo y abuso del poder. Ello generó una sociedad poco participativa y poblada, en su mayoría, de individuos anárquicos que negociaban su espacio vital con los personajes políticos en detrimento del Estado y las instituciones. La ciudadanía en México estuvo a punto de desaparecer por la generalizada desobediencia a las leyes, la estructura social y el orden moral. La corrupción, el cinismo de la autoridad, el cacicazgo, la impunidad... han causado que los gobernantes sean vistos como personas nefastas, lejos de ser líderes ejemplares e influyentes sobre la población.

Esta forma particular de dominación en México se nombró "corporativismo" y generó un monopolio político que se concentró en el Partido Revolucionario Institucional (PRI) en la mayor parte del siglo XX. El corporativismo y el clientelismo como formas de convivencia desarrollaron un "Estado anómico" que garantizó la estructura autoritaria en que la sociedad se desarrollaba. El régimen de la Revolución Mexicana perduró, al menos, setenta años, porque gozó de una amplia base de legitimidad social.

Los grupos inconformes se mantuvieron al margen de la participación, pero al avanzar la descomposición del régimen revolucionario lograron intervenir en el contexto político; en ocasiones con éxito y la mayoría de las veces con un fracaso cargado de represión. La historia del sistema político y la transición democrática en México dan cuenta de ello. Uno de estos sectores históricamente inconformes han sido los estudiantes universitarios: los vasconcelistas en 1929, los progresistas en 1968 y el movimiento “\#YoSoy132" de 2012. Estas participaciones de los jóvenes universitarios son representativas de los intereses de la clase media.

El argumento central del presente trabajo sostiene que aun cuando, por muchas décadas, el sistema político mexicano se caracterizó y adquirió estabilidad gracias al corporativismo y al clientelismo, estos principios deben ser sustituidos

* Los autores agradecen a César Cansino y Samuel Schmidt su colaboración para la elaboración de este artículo. 
por otros medios y prácticas si se piensa en la modernización y democratización real. Es decir, bien sea la ruta liberal o la social, la clase media y su afán de integrarse a la modernidad, la globalización, el "Faculty Club", la "Cultura de Davos" o la "Cultura de Porto Alegre", exigen la democracia en su estilo de vida.

El corporativismo brindó una alta institucionalidad al sistema político en su conjunto. Políticos, empresarios, obreros y la sociedad en general participaban dentro de las organizaciones, canalizando sus demandas a través de las instituciones establecidas, y aceptaban la estructura del sistema como legítima. Esta estructura tenía como objetivo principal el control del poder político y no la generación de participación política. El clientelismo permitía la comunicación de la sociedad con las instituciones corporativas; así se decidían las políticas gubernamentales y las elecciones locales, estatales y nacionales.

La verdadera democratización de México pasa por anular el poder de los monopolios públicos y privados, así como del clientelismo político. La democracia y la modernización del país adquieren visos reales y asequibles siempre y cuando se haga frente al corporativismo, concepción política susceptible de ser suplantada por otra más moderna y eficaz.

Con este propósito, resulta significativa la idea de interpretar los intereses de la clase media a través de los jóvenes universitarios. La clase media, situada entre la opresión de los dos polos corporativistas, tiene que mantener a los unos y a los otros. Es una clase que trabaja, que paga impuestos, que tiene que estudiar y que, paradójicamente, es ignorada constantemente por la definición de la agenda pública en beneficio de los poderes fácticos "de arriba" y "de abajo". La clase media es la inteligencia del país. De ella depende el éxito de la modernización y de la democracia mexicana; empero, sus ideas son incapaces de encontrar reflejos en la realidad, y cuando se empeña en hacerlo ha sido sometida y reducida a dos opciones: elegir el clientelismo de los pobres o el de los ricos, dejando siempre -teniendo que dejar- de ser ella misma. El hecho de que la clase media haya tenido que supeditar su interés al de los consorcios públicos y privados responde a que estos últimos están organizados para presionar la formulación de la agenda política en su favor, mientras que la clase media adolece de esta cualidad fundamental para representar, mediante su cohesión grupal, un peso específico relevante para la presión social.

En otras palabras, la clase media en México es la más consciente de los problemas sociales, económicos y políticos. Su representación mediante el sector estudiantil universitario como un grupo de presión para incidir en la formulación de la agenda política nacional, pone de relieve su capacidad de organización e 
influencia como sociedad civil, lo cual puede mostrarse a través del uso de la tecnología del internet. ${ }^{1}$

La internet supone una herramienta poderosa para la comunicación de los intereses de la clase media y para la adhesión grupal anhelada, pues las posibilidades mediáticas e informativas han hecho desaparecer toda distancia temporal y espacial entre las personas que comparten esta necesidad de formar una agrupación ciudadana al margen de todo partido político, y hacer sentir su peso y opinión.

\section{Los estudiantes universitarios como sujetos de clase media}

Definir la clase media desde las ciencias sociales entraña una enorme dificultad. Como dice Zamorano, "es como tratar de atrapar una anguila que se nos escapa de las manos". La investigadora cita en dicho artículo los enfoques de los siguientes autores: EsCOBAR y ROBERTS, quienes la clasificaron, preeminentemente, de acuerdo al nivel de ingreso y la profesión, seguidos por la escolaridad y el estilo de vida; LOAEZA, quien considera a la educación como base de su identidad, una identidad que deviene en modos de comportamiento, patrones de consumo y códigos de diferenciación simbólica, y TARRÉs, que parte de sus espacios de residencia como reveladores de un estilo de vida y una aproximación a la vida política.

Para Zamorano, no obstante, si bien estos criterios pragmáticos coadyuvan a la aprehensión de este particular objeto de estudio, no resuelven algunas paradojas elementales (como el hecho de que un próspero agricultor tenga ingresos mayores a los de un profesionista asalariado), por lo que prefiere recurrir a la sentencia del periodista norteamericano FrIEDMAN: "ser de clase media es un estado del alma, no una situación de ingresos".

Y ese estado del alma, en el caso de la clase media mexicana, quedó inmejorablemente plasmado en la obra de Gabriel CarEAGA. ${ }^{3}$ El sociólogo precisa, entre otras cosas, fundamentalmente dos: 1) que este heterogéneo grupo (constituido por burócratas, empleados, pequeños comerciantes, profesionistas, intelectuales, estudiantes, técnicos, gerentes de banco, secretarias...) liga al proletariado con la burguesía, y 2) se mueve "dentro de un mundo de mistificación, de ilusiones, de

\footnotetext{
1 Cansino, C. "Comentocracia vs. tuitercracia", columna semanal en Política para ciudadanos, disponible en: http:// politicaparaciudadanos.blogspot.mx/2012_05_01_archive.html.

2 Zamorano Villarreal, C. C. "Los hijos de la modernidad: movilidad social, vivienda y producción del espacio en la ciudad de México", en Alteridades, México, vol. 17, No. 34, julio-diciembre de 2007, pp. $75-91$ (disponible en: http:// redalyc.uaemex.mx/ src/inicio /ArtPdfRed.jsp?iCve=74711468006).

${ }^{3}$ Careaga, G. Mitos y fantasías de la clase media en México, Joaquín Mortiz, México, 1974.
} 
XÓCHITL PATRICIA CAMPOS LÓPEZ / DIEGO MARTÍN VELÁZQUEZ CABALLERO

sueños desaforados, de frustraciones constantes, de sentimentalismo creciente". Careaga emite así su tesis principal: el mundo de la clase media es ideológico, "está mistificado". Para explicar esta mistificación, CAREAGA se remonta a la Colonia y halla las raíces históricas de esta clase en los criollos, quienes sufrían desde entonces el desgarramiento social de "querer ser y no poder" y la inconformidad frente a la repartición de la riqueza, motivos que impelieron el deseo de cambiar el orden jurídico-político y económico-social mediante un movimiento independentista, orientado, desde luego, a la obtención del poder y, consiguientemente, una mayor riqueza.

No resulta extraño, pues, que CAREAGA ${ }^{4}$ nos haga conscientes de que esta clase $\mathrm{y}$, más concretamente, sus jóvenes han evolucionado, haciéndose más críticos, politizados y activos en la vida política y social. El movimiento estudiantil de 1968 -señala CAREAGA - es un claro y palpable efecto de este desarrollo.

En la visión del sociólogo, la clase media decaería paulatinamente hacia un mundo banal, especialmente durante el porfiriato, gastando su dinero en un esfuerzo imitativo tan constante como infructuoso por hallar su sitio entre el prestigio, la seguridad y el bienestar que imputa a -o del que goza efectivamente- la alta sociedad.

CAREAga ${ }^{5}$ aborda exhaustivamente otros temas que conforman la descripción integral de esta clase, tales como su forma de vivir -y mistificar- el erotismo, el ocio, etcétera. Para los fines argumentativos pertinentes a este trabajo, sin embargo, creemos que basta con apreciar a este grupo social con cuanto hasta aquí se ha dicho: se trata de un grupo heterogéneo que históricamente ha existido en el querer ser, y tener que ceder ante los intereses de otros grupos más organizados o pudientes, de arriba y de abajo, a los que, de alguna forma, vincula. Su juventud está ilustrada y politizada, y su forma común de organización o inserción en la sociedad es la familia.

A todo esto cabe agregar un hecho sociológico evidenciado recientemente por el analista Jorge G. CASTAÑEDA en su obra Ex Mex: From Migrants to Immigrants:

La nueva clase media mexicana proviene de la economía informal, de nuevas industrias o de nuevos servicios; esta clase se ha concentrado menos en la Ciudad de México y, cultural y socialmente es más 'rougher-edged' (agreste), así como más morena, de baja estatura y parecida al resto de la sociedad mexicana. ${ }^{6}$

\footnotetext{
4 Idem.

5 Idem.

${ }^{6}$ CASTAÑEDA, J. G. Ex Mex: From Migrants to Immigrants, Nueva York, The New Press, 2008, disponible en: http://books. google.com.mx/books?id=fvB1AAAAMAAJEtq=jorge+casta\%C3\%B1eda+ex+mextdq=jorge+casta\%C3\%B1eda+ex
} 
Posteriormente, CASTAÑEDA continúa con este nuevo análisis sobre la clase media de países emergentes, apuntando que la premisa detrás de esta afirmación es que "en muchas de las llamadas sociedades emergentes, la clase media ha crecido a lo largo de los últimos 15 años, para alcanzar ya casi la mitad de la población en países como China, Brasil, Chile, México, y dentro de 10 o 15, la India”. En esa misma publicación ${ }^{7}$ el analista recurre a una definición "un poco heterodoxa" de clase media, formulada por The Economist a partir del análisis reciente de especialistas chinos, hindús y el Banco Mundial.

The Economist considera tres factores: a) qué y cómo consume; b) el hecho de que tras asegurar sus necesidades básicas (vivienda y alimentación), exige que le sobre cerca de un tercio de su ingreso para gastos discrecionales, y c) disponer de un ingreso de entre 10 y 100 dólares diarios.

CASTAÑEDA agrega a estos criterios uno más, "quizás más simplista y clásico -aclara- pero no menos sugerente", y lo hace en virtud de que "no es lo mismo acceder a los niveles de consumo de clase media en Manhattan, que en Chennai, Huangzhou, Porto Alegre o Chilpancingo", por cuanto la calidad del bien variará en concordancia con la del entorno, aunque esencialmente se refiera a lo siguiente: adquirir, consumir y poseer bienes y servicios tradicionales de clase media, tales como una vivienda digna, aunque pequeña; un automóvil; acceso a crédito; el conjunto de bienes de consumo duradero (televisión, refrigerador, lavadora de ropa, computadora, teléfono fijo o celular, etcétera); vacaciones anuales, por modestas que sean; acceso a salud y educación pública o privada, buena o mediocre, pero que permite una cierta certeza y movilidad social.

Tras haber puntualizado estos criterios, el analista esclarece el porcentaje que en el México de hoy corresponde a éstos, grosso modo: entre el 60 y el 65 por ciento de los 26,541,327 hogares de acuerdo con la Encuesta Nacional de lngresos y Gastos de los Hogares 2006 (ENIGH) del Instituto Nacional de Estadística y Geografía (INEGI).

Así, si México cuenta con 110 millones de habitantes, y de ésos, 40 millones son pobres, entonces 70 millones no lo son, y si no son pobres pertenecen en su mayoría a una clase media, vieja o nueva, baja o media, del norte y del centro, o incluso del sur: sean los nuevos sectores globalizados, o los petroleros de Ciudad Madero de la vieja economía protegida. Es decir, el grupo en cuestión, hoy por hoy, asciende a 70 millones de habitantes, independientemente de su nueva o vieja pertenencia a dicha clase o cualquier otro distintivo.

+mex\&tsource $=$ bl\&tots=M8jANEvPgG\&tsig=|gF1qGCIRbeWbPiWOaGM5ty4tJOEth $\mid=$ en\&tsa $=$ XEtei $=$ jwNAU0a8L8iq20X mwoGYDQCtved=0CC406AEwAO.

7 ldem. 
XÓCHITL PATRICIA CAMPOS LÓPEZ / DIEGO MARTÍN VELÁZQUEZ CABALLERO

Hasta aquí podría vislumbrarse ya la enorme resonancia que podría tener la cohesión de un grupo de clase media con miras a incidir políticamente, pero hay más: de acuerdo con la ENIGH, los seis deciles superiores en México -continúa Castañeda $-^{8}$ entran en el criterio de The Economist. El sexto, el más bajo de éstos, tuvo en 2006 un ingreso diario por persona de 180 pesos, esto es, con el tipo de cambio vigente en aquel momento, casi 20 dólares diarios. Se entiende que los cinco deciles siguientes tuvieron más ingreso (es un cálculo sencillo para transformar los datos de ingreso de la encuesta en cifras equivalentes a los criterios de la revista). En el apartado sobre el gasto, a partir del sexto decil, los mexicanos dedicamos el 30 por ciento o más del ingreso a gastos discrecionales, como bienes de consumo, automóviles..., pero también a mejorar la salud o la educación. Este decil, y quizás el quinto y el cuarto también, corresponden a lo que The Economist y nosotros podríamos llamar clase media en desarrollo, que hoy, de acuerdo con el economista hindú Surjit Bhalla, ya representa el 57 por ciento de la población mundial.

Para terminar esta reflexión, conviene tener presentes dos conclusiones:

1) Un poco menos de las dos terceras partes de la sociedad mexicana ya pertenecen a esa clase media -antigua o en desarrollo-.

2) En la ENIGH, sólo el 1 por ciento de los mexicanos se autodescribió como rico; el 4 por ciento, como perteneciente a la clase media alta; el 44 por ciento a la clase media, y el 34 por ciento a la clase media baja.

Como se ve, nadie realmente se asumió como pobre, de cuanto sale a relucir que, aspiracionalmente, todos o casi todos los mexicanos se entienden a sí mismos como pertenecientes a la clase -o clases- media. Un dato por demás interesante si se piensa en la resonancia que podría tener un grupo cohesionado bajo esta denominación.

\section{Los estudiantes universitarios y sus preferencias electorales (el caso de la BUAP)}

En el proceso electoral 2012 para elegir presidente de la República, diputados federales y senadores, se realizó una encuesta por la Escuela de Sociología y Ciencias Políticas de la Facultad de Derecho y Ciencias Sociales (FDCS) de la Benemérita Universidad Autónoma de Puebla (BUAP)..$^{9}$ Además de conocer el perfil del

8 Idem.

9 Facultad de Derecho y Ciencias Sociales. Encuesta 2012 sobre cultura politica en los estudiantes universitarios de Ia BUAP, FDYCS-BUAP, Puebla, publicada en el periódico La Jornada de Oriente, mayo de 2012. 
elector universitario, sus tendencias electorales, etcétera, se buscaba aproximarse a su cultura política. Para este ensayo también se tomó en cuenta la encuesta realizada por alumnos de la Escuela de Ciencias de la Comunicación (сісом) de la BUAP. ${ }^{10}$ Cada proyecto encuestó, por separado, a 700 y 500 alumnos, respectivamente, de diferentes facultades. La BUAP tiene una población estudiantil cercana a los 60 mil individuos.

De ambos trabajos es importante resaltar los siguientes datos: aproximadamente el 54 por ciento manifestaba abiertamente una intención de voto, mientras que el 34.4 por ciento se mantenía en duda. La preferencia política que los estudiantes favorecieron fue la de la izquierda: en ambas encuestas, el 53 (FDycs) y el 57 por ciento (сісом) de las preferencias electorales beneficiaban a Andrés Manuel López Obrador. El resto de las preferencias se dividían entre Enrique Peña Nieto, Josefina Vázquez Mota y Gabriel Quadri.

La condición del género no modificó las tendencias electorales. Empero, hay otros criterios que vale la pena resaltar. En la encuesta de la Escuela de Ciencias de la Comunicación se encontró que aproximadamente el 30 por ciento de los universitarios trabaja, y ello condiciona también su preferencia política: votan en función de quien represente condiciones laborales más favorables para ellos, así como mejora económica. En la encuesta de la Facultad de Derecho se denota una tímida secularización del pensamiento político de los jóvenes (30 por ciento aproximadamente) y una clara orientación ideológica de centro izquierda.

Los universitarios son un clivaje en la estructura social de México por su pertenencia a la clase media. Los resultados nos muestran los valores de un grupo que es progresista, moderno y que considera la igualdad social como una condición sine qua non de la consolidación política del país.

En 2006 la clase media se mantuvo al margen del apoyo a la izquierda; de hecho, su voto benefició al PAN, lo que permitió que las elecciones no se anularan y el régimen derechista se mantuviera en la dirección de la transición política.

Para 2012 las circunstancias fueron distintas. Los doce años de presidencia panista condujeron al país hacia el Estado fallido. Sus resultados en políticas de seguridad y bienestar social han sido un fracaso y ello motivó que las preferencias políticas de la frívola clase media ahora se condujeran a la izquierda.

Las elecciones presidenciales de 2012 mostraron otra división del país que también afloró en 2006. Roger BARTRA ${ }^{11}$ afirmaba que las elecciones representaban una polarización de los clivajes sociales que se manifiestan en la estructura social del país. La transición fallida puso en un dilema a la sociedad mexicana. 0

\footnotetext{
${ }_{10}$ Facultad de Ciencias de la Comunicación. Encuesta 2012. El voto y los jóvenes, FCCom-BuAP, Puebla, publicada en el Periódico de la FCcom, El Ahuizotito, vol. 1, No. 24, 10 de mayo de 2012.

1 Bartra, R. La fractura mexicana, Debate, México, 2009.
} 
se brindaba la oportunidad a la izquierda mexicana o se retornaba al viejo régimen. Se elegía la modernidad o la tradición. Estas opciones estuvieron representadas por el electorado mexicano en su mayoría. Las clases populares eligieron el retorno del PRI, mientras la clase media impulsó a la izquierda. A este sector pertenecen los jóvenes universitarios.

Estos conflictos sociales se han manifestado en forma radical durante varios periodos de la historia mexicana. Pueden ser vistos como una lucha de clases, ${ }^{12}$ una argentinización, ${ }^{13}$ o bien como el producto de un multiculturalismo que no termina de resolverse. ${ }^{14}$ Lo cierto es que la clase media, y particularmente los jóvenes universitarios, han sido más demócratas y modernizadores en la lucha por la historicidad. ${ }^{15}$ Se denomina así a la lucha por el papel en las relaciones sociales de producción, políticas y culturales, al trabajo de autoproducción de la sociedad, a la capacidad de intervenir en su propio funcionamiento, de producir sus orientaciones normativas, de desarrollo de la intersubjetividad y de construir sus prácticas en un momento determinado de su historia.

\section{Internet como instrumento de liberación}

La tecnología representó en esta elección un instrumento de libertad. Su importancia para la creación de redes sociales resulta pertinente en el esfuerzo por cohesionar a los jóvenes universitarios que tienen acceso a dicho medio y que lo utilizan de manera asidua, ya para el trabajo o el ocio, lo cual implica, en primera instancia, que es un grupo que sabe, en efecto, operarlo. La creación del movimiento "\#YoSoy132" se planteó como un producto netamente universitario. Sorprendentemente no fue en las universidades públicas sino privadas donde surgió. Pero, sobre todo, su nacimiento fue en las redes sociales de internet. Sostiene César Cansino: ${ }^{16}$ la democracia se hace en Twitter.

Como se ha dicho, los jóvenes universitarios son un grupo ilustrado que puede entender el contenido de la política, que comprende textos, que sabe

212 manejar una computadora. Su posición y estatus social les brinda la posibilidad de incidir políticamente mediante una acción de bajo costo: inscribirse en el grupo o red social.

12 GILly, A. La revolución interrumpida, Era, México, 1974.

${ }^{13}$ Camacho Solis, M. "Los nudos históricos del sistema político mexicano", en Foro Internacional, México, vol. 17, No. 4, octubre-diciembre de 1977.

${ }_{14}$ Garcia Cancuni, N. Culturas hibridas. Estrategias para entrar y salir de la modernidad, Grijalbo, México, 1994.

${ }_{15}$ Touralne, A. ¿Podremos vivir juntos?, FEE, México, 2000.

${ }^{16}$ Cansino, C. "Comentocracia vs. tuitercracia", op. cit. 


\section{A manera de conclusión}

Los jóvenes universitarios y la internet significaron la aparición de la cultura y cibernética en la política mexicana. La internet generó la formación de un grupo representativo de los intereses de la clase media que, gracias a su capital social, tomó la delantera en la definición de la agenda pública. Su objetivo fue el despegue de la clase media frente a la imposición y componenda de la partidocracia y los poderes fácticos.

El resultado de las elecciones nos muestra que las demandas modernizadoras y progresistas de la clase media serán coartadas triste y apriorísticamente, como siempre, por las empresas privadas avasalladoras del mercado y por los sindicatos públicos; es decir, por los monopolios estatales y particulares, que los comprometerán de antemano a salvaguardar sus intereses.

Esta confabulación inicial representará, una vez más, la ruina de la clase media mexicana. Los oprobiosos tratos impedirán otra vez, ivaya tragedia!, la creación de las condiciones sociales mínimas indispensables en que nuestra estructura social podría ofrecer a nuestra clase media una verdadera oportunidad para crecer económicamente.

Los jóvenes universitarios, como puede verse en los resultados de las encuestas, como puede comprobarse en la historia, como lo señalan los estudios de política comparada, tienen una nostalgia del futuro, un anhelo de modernidad. Su organización en movimientos sociales muestra su alistamiento para subsanar la disfunción primigenia de la estructura social imperante en México mediante la creación de un grupo que pugne por una sociedad antimonopólica, que desarticule los consorcios privados y estatales mediante las, así llamadas, particiones, liberando con ello verdaderamente la economía en el ámbito privado, ampliando así las alternativas para una clase media que reivindica su derecho a la movilidad social, en vez de resignarse a ser esclava de esos contubernios.

La perspectiva de los jóvenes universitarios tomará la delantera, si no en el gobierno, en el ámbito académico y de la opinión pública libre. Los universitarios encarnan fidedignamente las legítimas aspiraciones de un gran sector social capaz de instrumentar los cambios que reclama con presteza la consolidación democrática; esto es, una democracia creadora de las condiciones necesarias para el despegue de la clase media, no de cuanto imponga, como siempre, dicha componenda entre la partidocracia y los poderes fácticos, entre el corporativismo y el clientelismo.

Frente al futuro, México tiene una lista de pendientes a realizar. Uno de ellos -sin duda el mayor- es el establecimiento de un Estado de derecho y respeto a 
XÓCHITL PATRICIA CAMPOS LÓPEZ / DIEGO MARTÍN VELÁZQUEZ CABALLERO

la ley, destinado a combatir la inseguridad, la impunidad, la corrupción, el narcotráfico y las conductas ilegales. Otro es la larga lista de medidas modernizadoras en los terrenos económicos: pensiones y salud, laboral, energética, fiscal, educativa, financiera, minorías, telecomunicaciones y registro de propiedad. A lo que hay que añadir la llamada reforma de Estado, cuyo objetivo es el de establecer reglas internas de gobierno que faciliten su operación y que en la práctica no ha servido de manera efectiva, puesto que los gobiernos estatales y municipales tienen su propia estructura operativa, la cual tiene que adaptarse a las exigencias federales, y en muchos de los casos hay incompatibilidad, haciendo más gravoso el trámite por parte del ciudadano.

Sólo la clase media y los jóvenes universitarios son conscientes del retroceso de la democracia en México. Definitivamente estamos regresando al pasado, al inicio del siglo xx, y por ello los jóvenes universitarios manifiestan la nostalgia por el futuro. La deuda con ellos debe pagarse antes de que opten por abandonar el país. 